\title{
Adopting a Digital Business Operating System
}

\author{
Jan Bosch \\ Chalmers University of Technology \\ Department of Computer Science and Engineering \\ Gothenburg, Sweden \\ Email: jan@janbosch.com
}

\begin{abstract}
The role of software in society and in industry in particular continues to grow exponentially. Most companies either have or are in the process of adoption continuous deployment of their software at products in the field and collect data concerning the performance of their systems. The continuous, fast feedback loops that companies now have available allow for a fundamentally different way of organizing. In fact, based on our work with dozens of companies, we have come to the conclusion that companies are moving towards a new, digital operating system. In this paper, we first present the key elements of the digital operating system and then discuss some of the challenges companies experience during the transformation.
\end{abstract}

\section{INTRODUCTION}

A S A POPULAR QUOTE says, in the future, all companies will be software companies. It is safe to say that this is no longer the future, but reality today. Ranging from telecommunications to automotive and from banks to retail companies, the key differentiator for virtually any company these days is it's ability to create, deploy and evolve software better than its competitors. In fact, every company these days is a software-intensive business (SIB).

One of the main focus areas for SIBs is the adoption of continuous deployment, meaning that new software is deployed in systems on a frequent basis (at least every agile sprint). As part of continuous deployment, the company often also deploys instrumentation to ensure that the systems continue to function as desired. This instrumentation generates data that not only provides information about any quality issues, but also about the value that the software is generating for customers and for the company itself. This causes the adoption of data-driven practices.

Generalizing from these observations, and based on research that we have conducted with dozens of companies over the last decade, we have concluded that industry is moving towards an new, digital business operating system. This operating system consists of four dimensions:

- Speed: The history of SIBs is defined by constantly increasing speed. From yearly to quarterly to continuous releases, software is deployed more frequently. The primary driver for this speed is the shortening of feedback loops. The goal is to shorten the time from making a decision to observing or measuring the effect of the decision to the shortest possible.

This work was supported by Software Center (www.software-center.se)
- Data: When a company has a mechanism to deploy new software to its servers or products in the field, this implies that it also is possible to get data back. Although the notion of "Big Data" is prevalent and especially online companies can be very advanced in their use of data, such as through the application of $\mathrm{A} / \mathrm{B} / \mathrm{n}$ experimentation, our research shows that many companies still make quite limited use of the available data as a resource.

- Ecosystems: The third dimension is concerned with the ecosystems surrounding a SIB. Although traditional companies tend to aim at performing as much of the required activities internally, modern companies focus their own resources on the activities where the company is uniquely differentiating and partner with others for everything else.

- Empowerment: Finally, when a SIB has a solid understanding of what it seeks to do in-house and the data to track the creation and delivery of value, the need for the traditional hierarchical organization disappears or at least is diminished radically. Instead, individuals and teams can be empowered to deliver on defined output metrics without having to be managed in conventional ways. Teams that deliver continue to thrive and teams that fail to deliver on expectations receive help and support to improve or, failing that, are disbanded.

The remainder of this paper is organized as follows. In the next section, we present each of the dimensions of the digital business operating system framework in more detail. Subsequently, we highlight some of the challenges that companies experience when adopting aspects of the framework. Finally, we conclude the paper in section IV.

\section{Digital Business Operating System}

As we discussed in the introduction, software intensive businesses tend to adopt aspects of a new digital business operating system framework. This framework consists of four dimensions, i.e. speed, data, ecosystems and empowerment. In the subsequent sections, we present each dimension in a more detail.

\section{A. Speed}

Companies evolve through a number of typical steps when increasing the speed of their operations. As shown in figure 1, in our research, we have identified five key steps. These steps are traditional development, agile development, continuous integration, continuous deployment and, finally, R\&D as an 


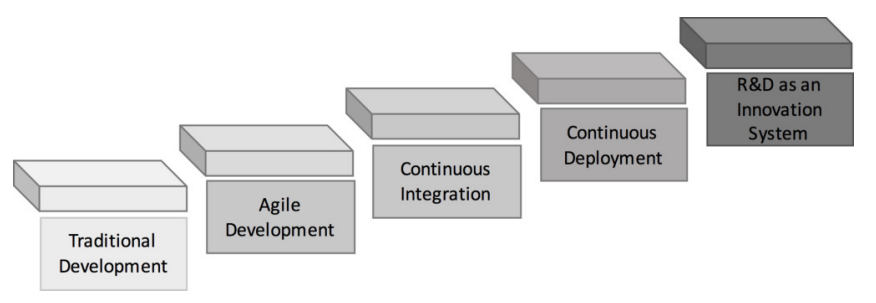

Fig. 1. The speed dimension

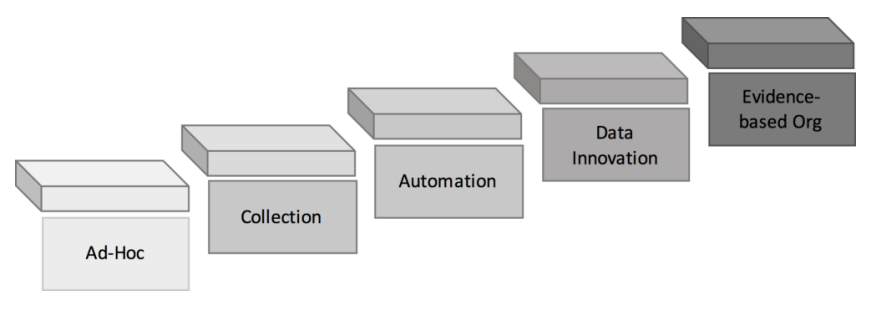

Fig. 2. The data dimension

experiment system. All except the last term are well known. The final step is where the company starts to use its installed base and data capability to experiment with alternative ways of realizing functions and features.

For more information on the speed dimension, we refer to [5] and [4].

\section{B. Data}

The data dimension also presents the typical steps that a company evolves through when adopting data-driven practices. As shown in figure 2, companies move from working with data in an ad-hoc fashion to automating the collection to data. The next step is where the company introduces dashboards and other techniques to automatically collect, analyze and present data. The data innovation step is where the company continuously looks for novel insights in the available data in order to continuously update and improve dashboards and other outputs. The final stage is where all processes in the company are driven by data, including hiring, performance reviews and management.

For more information on the data dimension, we refer to [6], [7] and [8].

\section{Ecosystems}

The third dimension is concerned with the way the company works with the ecosystems surrounding it. No company is an island and by necessity interacts with suppliers, customers, complementers, competitors, regulators, governments and other stakeholders. However, many companies have a tendency to focus their energy internally and try to conduct as much as possible inside the walls of the company. Companies that have adopted the digital business operating system are much more concerned about focusing their own resources where they have the most differentiation and, consequently, can add the most value. For everything else, these companies engage their ecosystem partners.

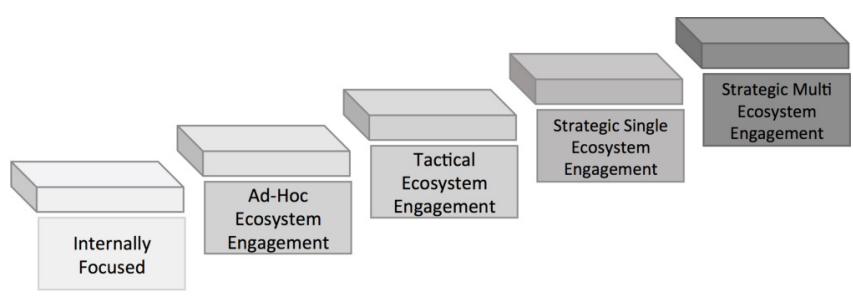

Fig. 3. The ecosystems dimension

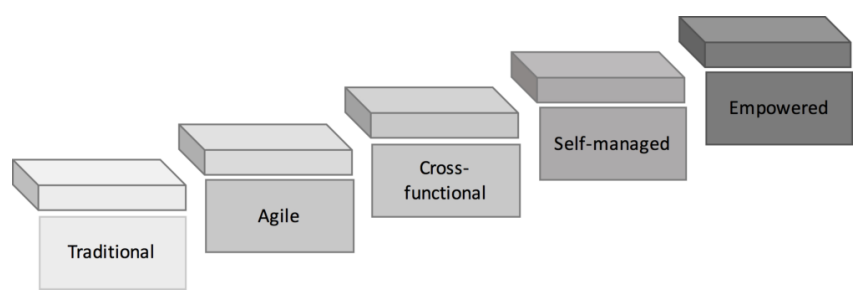

Fig. 4. The empowerment dimension

In figure 3 , the typical evolution path for a company is shown. Starting from an internal focus, the company initially engages with ecosystem partners in an ad-hoc fashion, then proceeds to a more tactical approach and over time becomes increasingly strategic about its engagements with the ecosystem.

For more information on the ecosystems dimension, we refer to [3], [10] and [2].

\section{Empowerment}

The final dimension of the digital business operating system addresses the organizational question. Traditionally, companies have been organized hierarchically, largely mirroring the military organizations that were one of the few examples of organizing large groups of individuals that existed at the beginning of the industrial revolution. Hierarchical organizations are effective for organizing repetitive and often manual work in low-complexity contexts, but fail in high complexity situations where creativity and non-standard solutions are required. As virtually all repetitive work these days is automated, the only work left for individuals is that which can not easily be standardized. Consequently, we need alternative ways of organizing and the common denominator is empowerment.

In figure 4, the typical evolution path for a company moving from traditional hierarchies to empowerment is shown. Starting from a hierarchical approach to organizing, agile software development provides a first step on the transition to empowerment as agile teams have significantly more freedom to operate than their counterparts working in a hierarchical waterfall approach. From there, we see companies adopt increasing cross-functional empowerment, fully self management and finally the fully empowered state.

For more information on the empowerment dimension, we refer to [9] and [1]. 


\section{Challenges}

Adopting the digital business operating system for a company that has worked traditionally is quite challenging as it affects all functions in the company. In addition, everything from business models to system architecture, from process to organization and from formal structures to company culture is affected. Consequently, the challenges that companies experience are significant and it is sometimes difficult to separate root causes from symptoms that are the consequence of root causes.

In order to provide a high-level overview of the key challenges that companies experience, we use the BAPO model [1]. The BAPO model considers business, architecture, process and organization. In the sections below, we briefly discuss the key challenges that companies experience when adopting the digital business operating system.

\section{A. Business}

Most product companies have traditionally maintained a transactional business model, meaning that customers engage with the company every couple of years or even decades. For instance, most people will buy a new car every five years and have little connection with the car company in the meantime. One of the consequences of digitalization is that it typically assumes a continuous deployment model for software. This has several consequences for the business.

The first consequence is that the business model changes from a product-centric to a service-centric model. In many industries, customers subscribe to a service in order to get access to the product, rather than buying it outright. That changes the customer relationship from a transactional to a continuous one. A second consequence is that it requires a different financial model because the company needs to finance the product for the customer and then earn back the cost through service.

\section{B. Architecture}

There are two primary changes required to the architecture when adopting a digital business operating system. First, because we're looking to support continuous deployment, it is typically required to deploy individual components independently in order to avoid significant downtime on systems in the field. This requires a significantly improved level of modularization.

Second, as we're looking to use the data from systems in the field for managing quality and for determining whether we're delivering value for customers, the architecture needs to be prepared for easy instrumentation of arbitrary parts of the system. Our research shows that companies often seek to add points of data collection and instrumentation continuously.

\section{Process}

Continuous deployment, as we saw in the speed dimension of the digital business operating system, requires continuous integration and test. Although many companies pride themselves on the high quality of their systems, experience shows that many companies require significant investment in build and test infrastructure before the quality of software reaches a point where it can be deployed continuously.

Second, although agile practices are widely adopted at the team level, true business agility where all functions in the company operate in short cycles and can rapidly respond to changes in the market is an elusive concept for many organizations. Product management, marketing, sales, systems engineering and general management often have a tendency to operate in yearly and quarterly cycles and convincing these functions to adopt a continuous, agile approach is a significant challenge.

\section{Organization}

As indicated in the discussion around the empowerment dimension, to successfully operate in this new approach, the traditional hierarchical and functionally structured company needs to be reorganized into smaller cross-functional teams. The amount of coordination overhead and delay in traditional organizations is simply too costly and slow.

An interesting observation from our research is that company culture often has significant number of implicit assumptions that are based on a waterfall style of doing business, meaning that the change towards digitalization requires a fundamental change of the company culture itself. As culture eats strategy for breakfast, as the saying goes, it requires significant effort and role modeling from leaders to instill the cultural changes required to successfully operate in this new model.

\section{CONCLUSION}

Virtually all companies these days are software-intensive businesses. Across domains, such as telecommunications, automotive, finance, defense and retail, the ability to create, deploy and evolve better software faster than competitors is the key differentiator.

Our research that we have conducted with dozens of companies over the last decade has led us to the conclusion that industry is moving towards an new, digital business operating system. This operating system consists of four dimensions:

- Speed: The history of SIBs is defined by constantly increasing speed. From yearly to quarterly to continuous releases, software is deployed more frequently. The primary driver for this speed is the shortening of feedback loops. The goal is to shorten the time from making a decision to observing or measuring the effect of the decision to the shortest possible.

- Data: When a company has a mechanism to deploy new software to its servers or products in the field, this implies that it also is possible to get data back. Although the notion of "Big Data" is prevalent and especially online companies can be very advanced in their use of data, such as through the application of $\mathrm{A} / \mathrm{B} / \mathrm{n}$ experimentation, our research shows that many companies still make quite limited use of the available data as a resource. 
- Ecosystems: The third dimension is concerned with the ecosystems surrounding a SIB. Although traditional companies tend to aim to performing as much of the required activities internally, modern companies focus their own resources on the activities where the company is uniquely differentiating and partner with others for everything else.

- Empowerment: Finally, when a SIB has a solid understanding of what it seeks to do in-house and the data to track the creation and delivery of value, the need for the traditional hierarchical organization disappears or at least is diminished radically. Instead individuals and teams can be empowered to deliver on defined output metrics without having to managed in a conventional way. Teams that deliver continue to thrive and teams that fail to deliver on expectations receive help and support to improve or, failing that, are disbanded.

The transition from a traditional mode of operating to this new operating system is far from trivial and in section III we discussed some of the key isseus that SIBs experience. These challenges range from business and business model to architecture, process, organization as well as the norms, beliefs and culture of the company.

In the context of Software Center, we continue to study the challenge of Digitalization with the partner companies and help accelerate the adoption of the digital business operating system at these and other companies.

\section{ACKNOWLEDGMENT}

I would like to thank the companies involved in the Software Center as well as other companies that I have interacted with during the last years.

\section{REFERENCES}

[1] Jan Bosch. Speed, data, and ecosystems: Excelling in a software-driven world. CRC Press, 2017.

[2] Jan Bosch and Helena Holmström Olsson. Ecosystem traps and where to find them. Journal of Software: Evolution and Process, page e1961, 2018.

[3] Petra M Bosch-Sijtsema and Jan Bosch. Plays nice with others? Multiple ecosystems, various roles and divergent engagement models. Technology Analysis \& Strategic Management, 27(8):960-974, 2015.

[4] Helena Olsson, Anna Sandberg, Jan Bosch, and Hiva Alahyari. Scale and responsiveness in large-scale software development. IEEE Software, 31(5):87-93, 2014.

[5] Helena Holmström Olsson, Hiva Alahyari, and Jan Bosch. Climbing the" stairway to heaven"-a mulitiple-case study exploring barriers in the transition from agile development towards continuous deployment of software. In 2012 38th Euromicro Conference on Software Engineering and Advanced Applications, pages 392-399. IEEE, 2012.

[6] Helena Holmström Olsson and Jan Bosch. From Opinions to DataDriven Software R\&D: A Multi-case Study on How to Close the "Open Loop" Problem. In 40th EUROMICRO Conference on Software Engineering and Advanced Applications, pages 9-16. IEEE, 2014.

[7] Helena Holmström Olsson and Jan Bosch. The HYPEX Model: From Opinions to Data-Driven Software Development. In Continuous Software Engineering, pages 155-164. Springer, 2014.

[8] Helena Holmström Olsson and Jan Bosch. Towards continuous customer validation: A conceptual model for combining qualitative customer feedback with quantitative customer observation. In International Conference of Software Business, pages 154-166. Springer, 2015.

[9] Helena Holmström Olsson and Jan Bosch. No more bosses? In International Conference on Product-Focused Software Process Improvement, pages 86-101. Springer, 2016.

[10] Helena Holmström Olsson and Jan Bosch. From ad-hoc towards strategic ecosystem management: the three-layer ecosystem strategy model. Journal of Software Evolution and Process, 29(7):e1876, 2017. 